\title{
Active hyperspectral mid-infrared imaging based on a widely tunable quantum cascade laser for early detection of plant water stress
}

\author{
Chaimae El Fakir $\odot$, a,* Maroun Hjeij, ${ }^{\text {a }}$ Ronan Le Page $\odot,{ }^{a}$ Luiz Poffo $\odot,{ }^{\text {a }}$ \\ Bastien Billiot, ${ }^{\mathrm{b}}$ Pascal Besnard $\odot,{ }^{\mathrm{a}}$ and Jean-Marc Goujon $\odot^{\mathrm{a}}$ \\ ${ }^{a}$ Université de Rennes, CNRS, Institut FOTON-UMR 6082, Lannion, France \\ ${ }^{\mathrm{b}}$ Agro Innovation International-CMI, Saint-Malo, France
}

\begin{abstract}
Plant water stress has been extensively studied using hyperspectral visible- and nearinfrared systems. Thermal imaging and the recent availability of widely tunable infrared quantum cascade laser (QCL) allow us to propose an active hyperspectral imaging system operating in the mid-infrared (MIR) band, where the system output consists of a series of narrowband subimages arranged across the reflectance spectrum of the sample, forming a hypercube data acquired by "staring" acquisition technique. To evaluate more precisely the capabilities of the active hyperspectral imaging, we propose a system composed of four powerful tunable QCL covering the 3.9- to $4.7-\mu \mathrm{m}$ and 7.5- to $11-\mu \mathrm{m}$ wavelengths ranges. Two cameras are used for detection: an InSb cooled camera ranging from 3 to $5 \mu \mathrm{m}$ and a bolometer from 7.5 to $13 \mu \mathrm{m}$ range. This system is validated by applying to growing plants for early water stress detection. Finally, we present and discuss results using partial least squares discriminant analysis classification technique to characterize water status of different plants, separated in two classes: control subjects were maintained at $80 \%$ of the amount of water to soil saturation ratio and stressed subjects at $20 \%$. Initial discrimination results have shown the efficiency of the proposed system. (C) 2021 Society of Photo-Optical Instrumentation Engineers (SPIE) [DOI: 10.1117/1.OE.60.2.023106]
\end{abstract}

Keywords: hyperspectral imaging; tunable quantum cascade laser; mid-infrared; spectroscopy; water stress; plant classification.

Paper 20201119 received Sep. 21, 2020; accepted for publication Feb. 3, 2021; published online Feb. 24, 2021.

\section{Introduction}

Plants are exposed to a constantly changing environment, which results in various biotic and abiotic stresses. Water stress is an abiotic stress that damages crop growth and productivity. ${ }^{1,2}$

Detecting water stress from the first symptoms is crucial to initiating crop irrigation to maintain plant development, but the symptoms are not visible to human eyes at first and when they become observable, plant health is already affected. ${ }^{3,4}$

Plant water stress is usually associated to with the measurement of soil moisture, measured using a tensiometer implanted in the soil to determine the force with which water is held. When the tension is high, it means that soil is dry. ${ }^{5}$

Recent studies show the detection of water stress in plants by measurements made directly from plants on different species such as potato, asparagus, grapevine, cherry, sorghum, and sunflower. ${ }^{4,6-8}$ Spectroscopy techniques ${ }^{9,10}$ and imaging systems ${ }^{11-13}$ were commonly used to determine the plant response to drought.

The spectral reflectance signature (the flux of reflected light) obtained by spectroscopy is based on the physical and chemical properties of the surface of the material examined. ${ }^{14}$ Appropriate spectral analyses can determine changes and can be used to identify the plant's physiological state and assess to water status. ${ }^{10}$ The visible region (VIS, 0.4 to $0.7 \mu \mathrm{m}$ ) includes a major portion of the information about the leaf reflectance as a result of the absorption of leaf

*Address all correspondence to Chaimae El Fakir, chaimaeelfakir16227@gmail.com

0091-3286/2021/\$28.00 ㄷ 2021 SPIE

Optical Engineering

023106-1

February 2021 • Vol. 60(2) 
pigments. ${ }^{15}$ This part of the spectrum is used to study changes in leaf pigments and is the basis of studies of plant health. ${ }^{16}$ The reflectance variation in the near-infrared region (NIR, 0.7 to $1 \mu \mathrm{m}$ ) is known as the red edge and is used to determine vegetation indices. Changes in these vegetation indices provide information about plant productivity, plant health, and stress, ${ }^{17}$ whereas the short-wave infrared (SWIR, 1 to $2.5 \mu \mathrm{m}$ ) is widely used in the evaluation of changes in leaf water content, particularly related to drought stress. ${ }^{18}$ The observations of leaves in the midinfrared (MIR, 2.5 to $11 \mu \mathrm{m}$ ) have less pronounced characteristics compared to the region between the VIS, the NIR, and the SWIR. The leaves were for a long time considered to be without characteristics in the MIR. With the recent availability of more accurate instrumentation for performing infrared measurements, studies have shown that spectral differences in this region can be caused by leaf features associated with the leaf structure, such as water content. ${ }^{6,19,20}$

Imaging has been shown to be effective in providing a reliable and quick non-destructive assessment of the water status in plants, ${ }^{21}$ including hyperspectral imaging that allows to extract simultaneously the spectral and spatial signatures related to the structure and to plant physiology, the result of which is a series of narrowband subimages arranged over the reflectance spectrum, forming a hyperspectral cube. ${ }^{13,22}$

In recent years, most hyperspectral systems have focused on VIS and NIR ranges due to their technological maturity and their various applications. ${ }^{23,24}$ Many studies showed the impact and the interest of using the infrared range for detecting early signs of stress in vegetation. ${ }^{11,21}$ This particular range appears to contain meaningful information on the features needed to discriminate samples, for example for contamination or quality inspection. ${ }^{18}$ The latest development of laser sources and imaging hardware allows us to access the hyperspectral MIR imaging in many new applications. ${ }^{25}$

In MIR wavelengths ranges, pushbroom (spatial scanning) ${ }^{26}$ are not commercially available for wavelength over $5 \mu \mathrm{m}$, but tunable source or filtering can enable staring (wavelength scanning) architecture.

Therefore, the focus of this paper is to unveil our current progress with our proposed hyperspectral imaging bench, for use in environmental monitoring applications. An optical architecture was proposed in 2019 by our laboratory. ${ }^{27}$ We are applying this imaging system to vegetation more precisely to a Helianthus annuus (sunflower), in order to keep track of the water status on the foliar surface. Our objective is to detect early plant stress in time, before irreversible damages and yield loss, in a non-destructive way.

In Sec. 2, we introduce our imaging system, based on recent tunable monochromatic light sources as MIR quantum cascade laser (QCL), and the image processing method to extract the reflectance spectra. In order to assess the system on vegetal stress detection, we propose a standardized daily stress application protocol. Section 3 presents our preliminary results: typical reflectance spectra are shown for each day of measurement, for stressed and reference samples. In Sec. 4, we propose a way to classify our plants and we discuss the results.

\section{Methods}

\subsection{System Configuration (Imaging System-Hardware)}

Our experimental bench is based on a system consisting of four tunable laser modules operating in MIR spectral range as shown in Fig. 1. The first QCL (QCL1), tuned from 3.9 to $4.7 \mu \mathrm{m}$, operates in pulsed mode, with a duty cycle quoted up to $10 \%$. Repetition rate can range up to $1 \mathrm{MHz}$, and the pulse width can be varied from 40 to $500 \mathrm{~ns}$. The maximum average output power is $11 \mathrm{~mW}$. The three other QCLs modules are: QCL2 tunable from 6.73 to $7.75 \mu \mathrm{m}$, QCL3 tunable from 7.69 to $8.64 \mu \mathrm{m}$, and QCL4 tunable from 8.39 to $11 \mu \mathrm{m}$. They operate in continuous mode with linewidths are $<10 \mathrm{~nm}$.

A mirror system enlarges the laser beam to $5 \mathrm{~cm}$ (diameter) in order to illuminate the sample and the Lambertian reference (gold plane) as shown in Fig. 2. The images acquired on the Lambertian reference are to be used to extract the incidence power of the laser beam generated.

The scattered flux is measured by two standard thermal cameras operating in different spectral bands. The first one is a cooled InSb camera (FLIR SC5000) operating in the 3- to 5- $\mu \mathrm{m}$ 


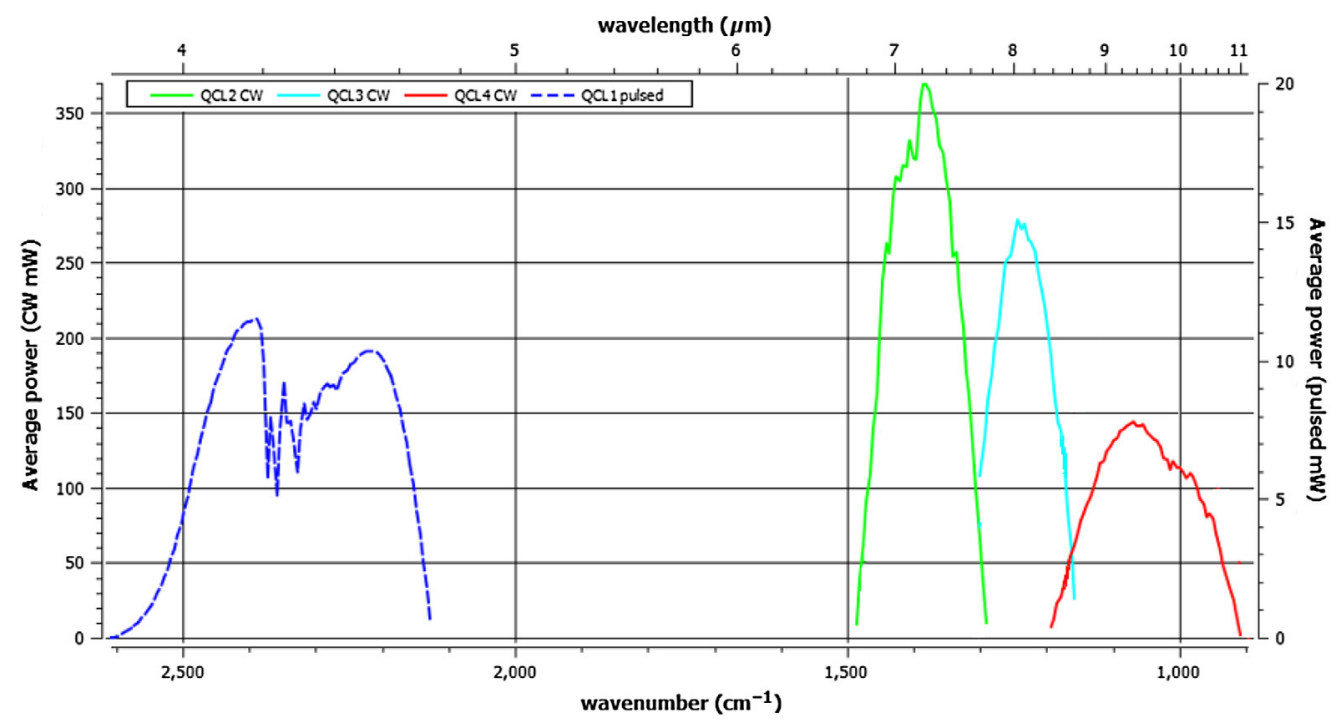

Fig. 1 Tuning curves of four QCLs, which provide a total tuning range of 3.9 to $11 \mu \mathrm{m}$.

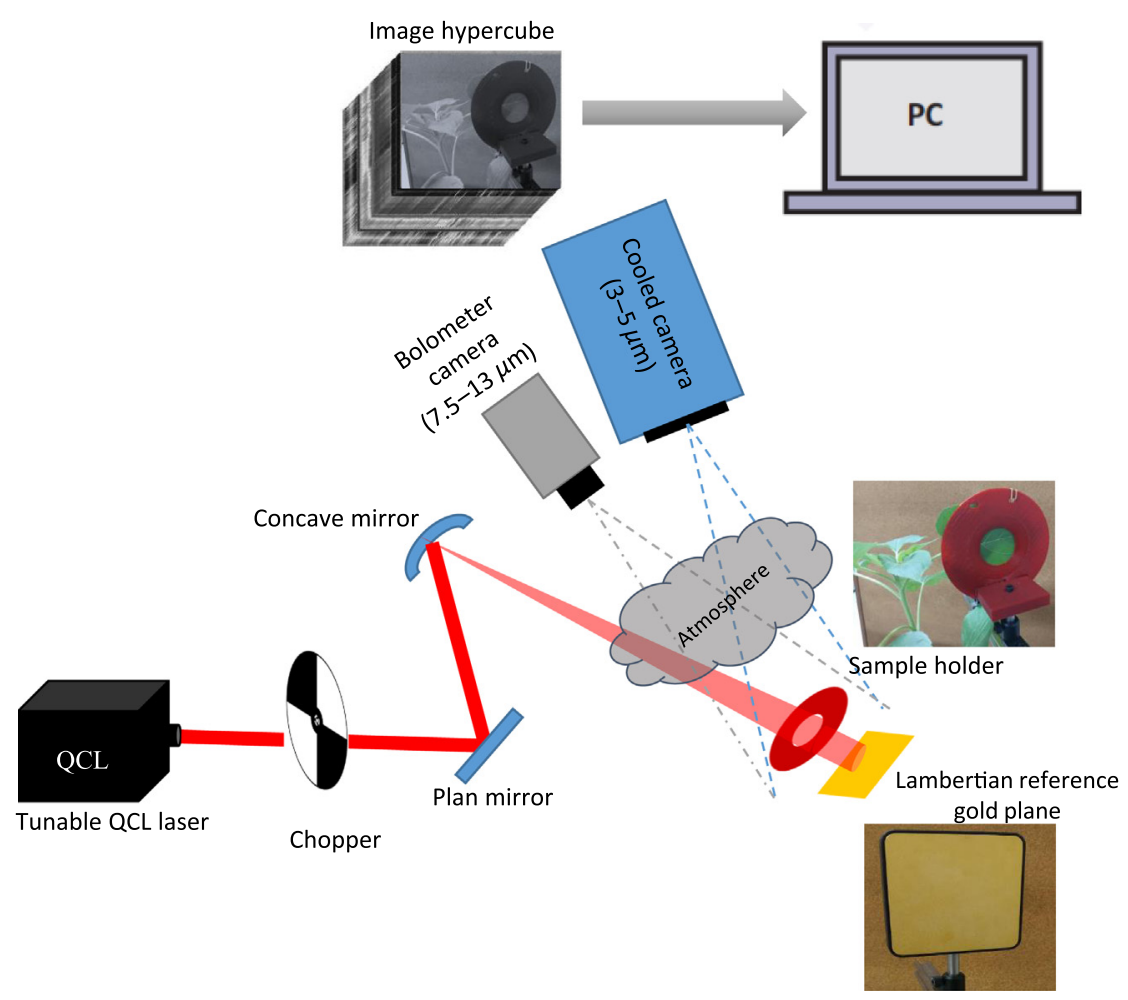

Fig. 2 Schematic of the experimental bench for active hyperspectral imaging.

range, with a focal plane array of $(320 \times 256)$ pixels of size equal to $30 \mu \mathrm{m}$ each pixel. The second one is a bolometric detector (FLIR A65) operating in the 7- to 11- $\mu \mathrm{m}$ range, with a spatial extent of $(640 \times 512)$ pixels of $17 \mu \mathrm{m}$ size.

The observation system performs a double acquisition (thermal background + laser signal) for each wavelength. This task was automated using a chopper synchronized according to the acquisition time of the two cameras: $700 \mu \mathrm{s}$ integration time for InSb camera and $20 \mathrm{~ms}$ for bolometric camera. A subtraction is performed between the image with laser illumination and without in order to eliminate the background of the scene as shown in Fig. 3. 
El Fakir et al.: Active hyperspectral mid-infrared imaging based on a widely tunable quantum...

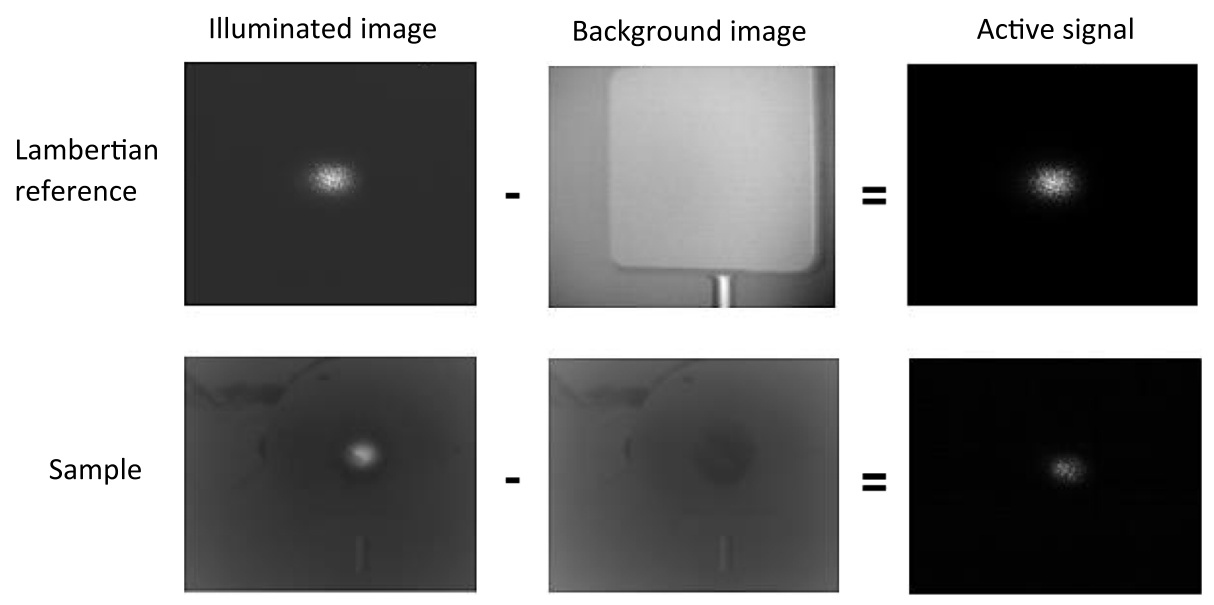

Fig. 3 Example of removing the background of the scene at $8 \mu \mathrm{m}$.

\subsection{Reflectance Spectrums Calculation from Acquired Images}

On the illuminated images, we notice the presence of the speckle effect due to the laser spatial coherence. To eliminate the effect of speckle on the measurements, a spatial average of the measurement area is applied. The spectral reflectance rate $\rho(\lambda)$ of the sample is obtained by the average of the intensity from the measurement area on the sample, normalized by the average intensity of the IR Lambertian (IRL) reference gold plane, which is expressed in this equation:

$$
\rho(\lambda)=\frac{\bar{M}_{\text {sample }}(\lambda)}{\bar{M}_{\mathrm{IRL}}(\lambda)},
$$

where $\rho$ is the reflectance rate between 0 and $1, \bar{M}_{\text {sample }}$ is the average illumination of the surface of sample, and $\bar{M}_{\text {IRL }}$ is the average of illuminated surface of IRL.

Figure 4 gives an example of the selection of measurement areas on images taken by the two cameras. An area of size $40 \times 30$ pixels was selected in the spectral range between 3.9 and $4.7 \mu \mathrm{m}$ and $150 \times 110$ pixels for wavelengths between 7.5 and $11 \mu \mathrm{m}$ in order to select the entire laser spot.

Two spectral resolutions are used ( $40 \mathrm{~nm}$ for 3.9 to $4.7 \mu \mathrm{m}$ and $50 \mathrm{~nm}$ for 7.5 to $11 \mu \mathrm{m}$ ) for a total number of 91 sampled wavelengths for each leaf measured. Table 1 is a summary of parameter settings.

\subsection{Plant Stress Protocol}

Our samples are Helianthus annuus (sunflower). Due to their dependency on ultraviolet rays from the sun like any other green plant, a growth chamber was established to fulfill their needs. Artificial light (LED lighting associated to a sodium lamp) was provided up to $18 \mathrm{~h}$ a day, and

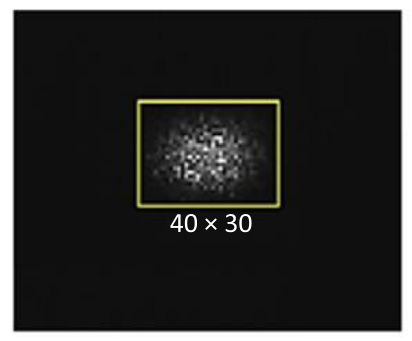

(a)

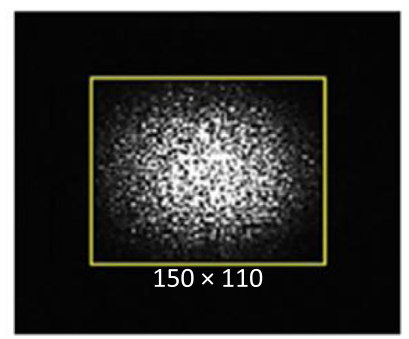

(b)

Fig. 4 Measurement areas in pixels: (a) spectral range between 3.9 and $4.7 \mu \mathrm{m}$ and (b) spectral range between 7.5 and $11 \mu \mathrm{m}$. 
Table 1 Parameter settings.

\begin{tabular}{lcc}
\hline \hline Specifications $(\mu \mathrm{m})$ & 3.9 to 4.7 & 7.5 to 11 \\
\hline Integration time $(\mu \mathrm{s})$ & 75 for IRL & Time constant $<8 \mathrm{~ms}$ \\
& 700 for leaf & \\
Spectral range $(\mu \mathrm{m})$ & 3.9 to 4.7 & 7.5 to 11 \\
Span $(\mu \mathrm{m})$ & 0.8 & 3.5 \\
Spectral resolution $(\mathrm{nm})$ & 40 & 50 \\
Number of sampled wavelengths & 20 & 71 \\
\hline \hline
\end{tabular}

turned off during the remaining hours in order to simulate natural light cycle. An extractor fan was used to regulate the temperature of the growth chamber. The growth chamber ensures same light and temperature for all plants.

In our series of ten plants, five are control subjects where the amount of water to soil saturation ratio is maintained at $80 \%(80 \%$ SWC) and five are stressed subjects with $20 \%$ SWC. The SWC is an important parameter in the plant hydrological cycle. ${ }^{28,29}$ This protocol is maintained during the 11 days of the experiment, when the plant stress becomes visible. We specify that the stress is applied on day 0 and the measurements started on day 1 .

Two leaves per plant at the same stem height have been studied, leading to a total of 3732 raw images a day (20 leaves $\times 91 \lambda+20 \times 91$ background +91 Lambertian +1 background). The measurements started every day at the same time and with the same plant and leaf sequence. This sequence was repeated every day to ensure the same measurement condition day after day for each leaf (same schedule). We alternated between stress and control subjects to prevent any bias that could be introduced due to the measurement duration.

Table 2 summarizes the quantity and timing of acquisitions.

\section{Results}

After 11 days of water stress application, plants under stress visually changed compared to the control subjects. Loss of leaf turgidity was observed in the plants on the 11th day of the experiment, as shown in the following pictures (Fig. 5).

\subsection{Reflectance Spectra}

We present in Fig. 6 the mean reflection rate spectra of the five control plants (blue curve) and the five stressed plants (red curve) during the 11 days of experiment. It is separated into two spectral ranges: 3.9 to $4.7 \mu \mathrm{m}$ and 7.5 to $11 \mu \mathrm{m}$ corresponding to each camera.

We notice that there are no significant difference between stressed and control plants. Reflection rate spectra are obtained over $8 \mathrm{~h}$ of measurement and each plant probably exhibits a different physiological state corresponding to its daily rhythm. This adds noise to the data and makes it impossible to accurately determine which wavelength is influenced by water stress. With more data, it would be possible to statistically find influential wavelengths to determine stressed elements. Another approach is to take all wavelengths of the reflection rate spectra and use then as a signature, even though there is a lot of noise, we can recognize the signature of water stress.

\subsection{Classification Images}

In order to validate the possibility of characterizing the water stress of growing plants at an early stage (before the stress indicators can be visibly detected), we propose to use a machine learning approach from the reflectance spectra of the plants. For a small amount of data, the most practical 
El Fakir et al.: Active hyperspectral mid-infrared imaging based on a widely tunable quantum...
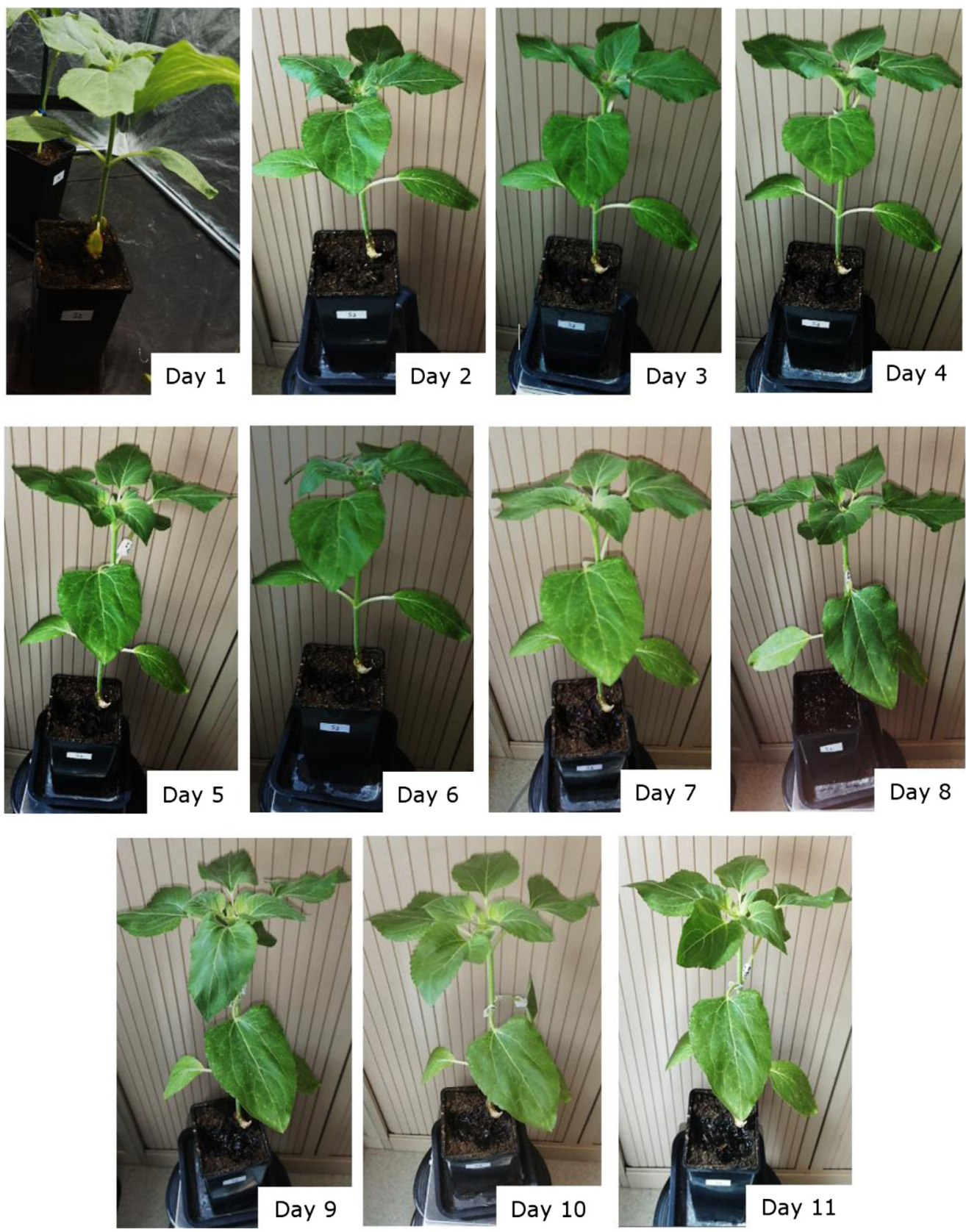

Fig. 5 Progression of water stress over time. Visible water stress is shown on 11 days of treatment.

Table 2 Samples quantity and acquisitions time.

\begin{tabular}{lcc}
\hline \hline Specifications & Control plants & Stressed plants \\
\hline Number of plants & 5 & 5 \\
Number of leaves by plant & 2 & 2 \\
Quantity of water $(\mathrm{g})$ & 836 & 209 \\
Acquisition days & 11 & 11 \\
Total number of reflectance images & 10,010 & 10,010 \\
\hline \hline
\end{tabular}


El Fakir et al.: Active hyperspectral mid-infrared imaging based on a widely tunable quantum...
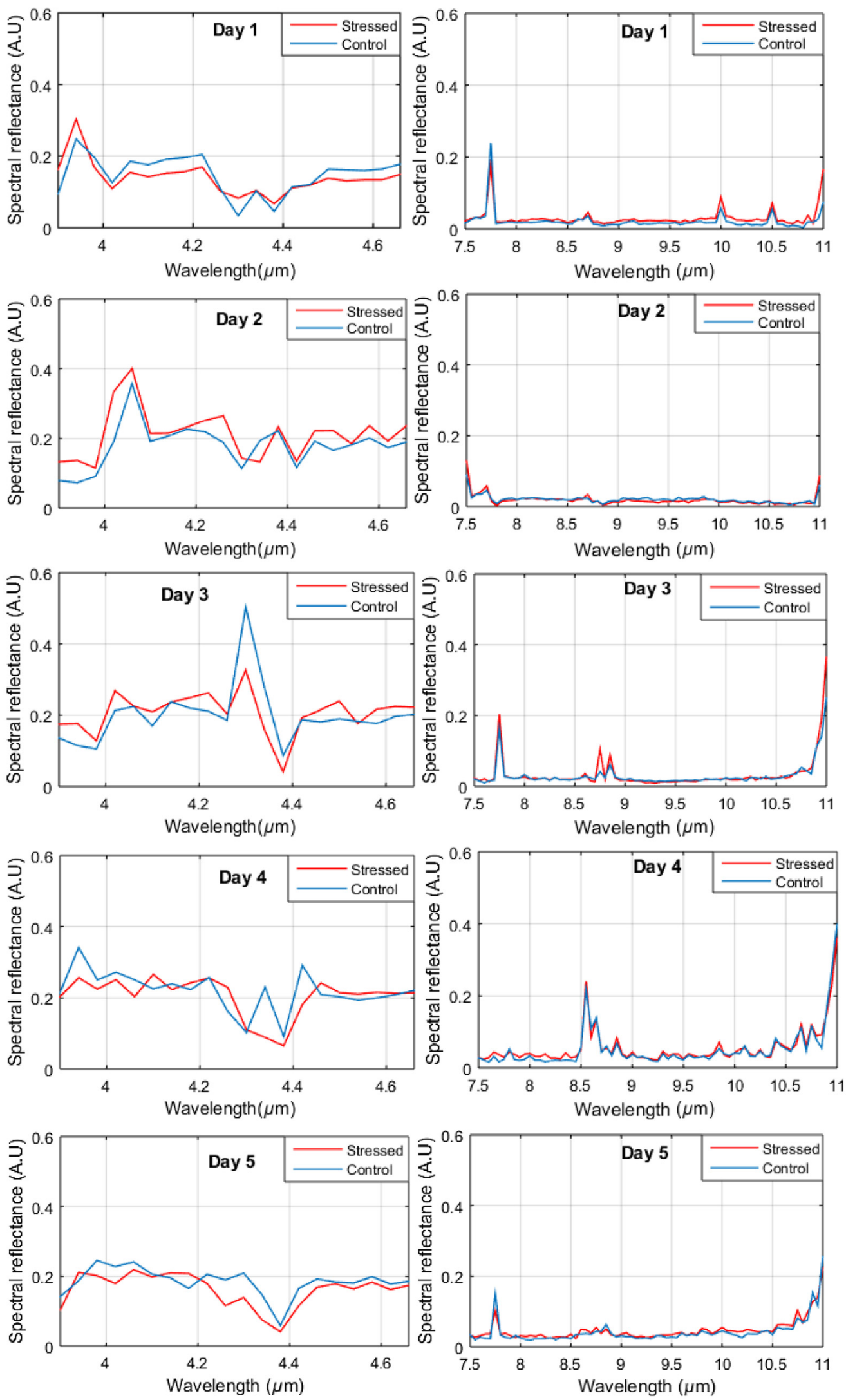

Fig. 6 Mean reflection rate spectra of control (blue) and stressed plants (red) during 11 days of experiment. 
El Fakir et al.: Active hyperspectral mid-infrared imaging based on a widely tunable quantum...
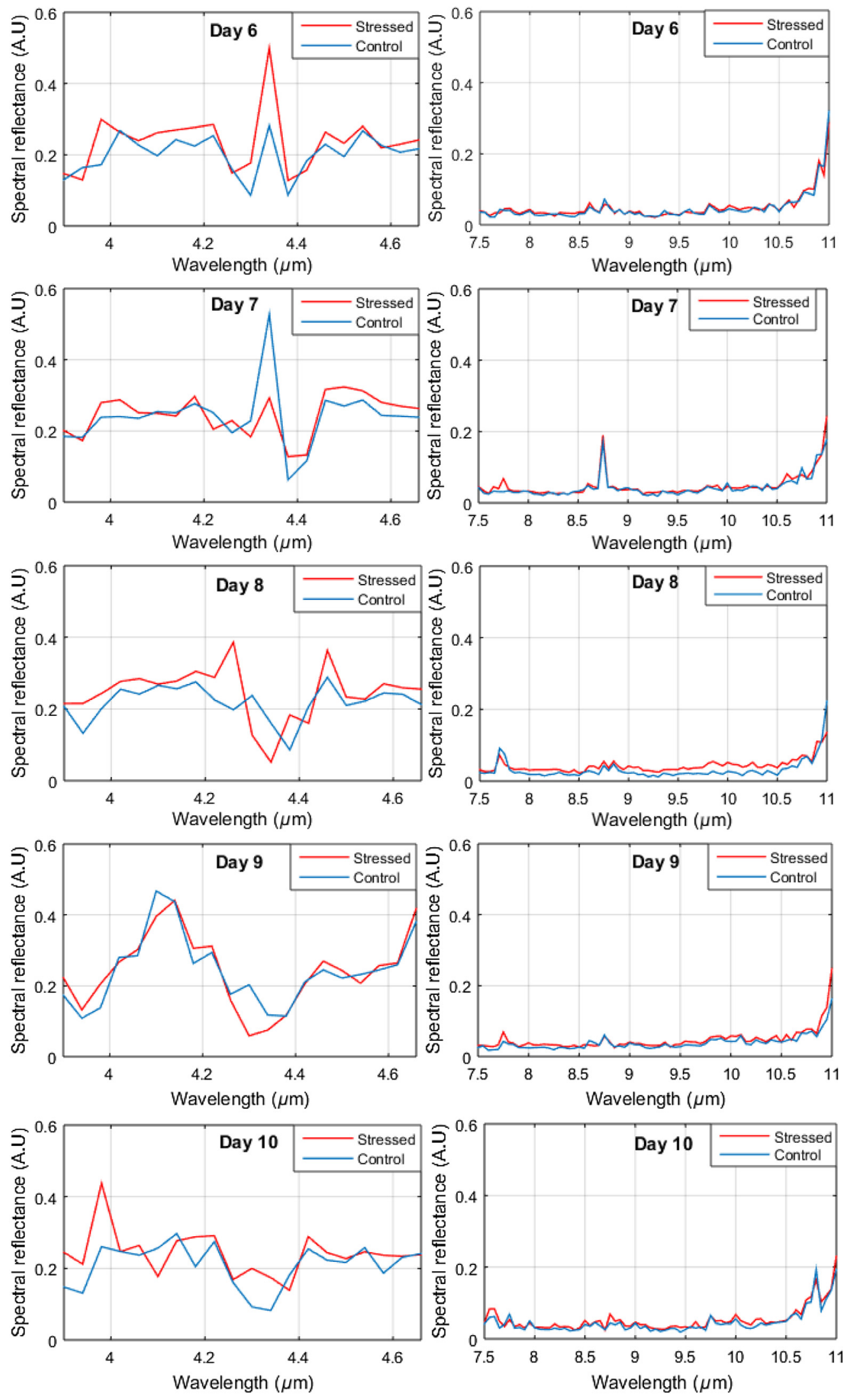

Fig. 6 (Continued.) 
El Fakir et al.: Active hyperspectral mid-infrared imaging based on a widely tunable quantum...
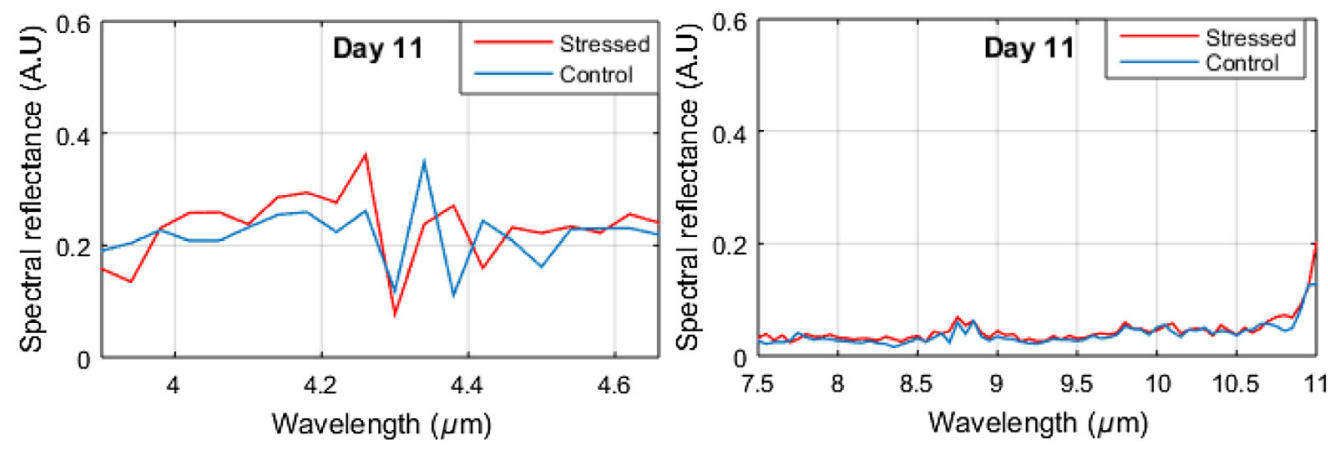

Fig. 6 (Continued.)

Table 3 Confusion matrix for binary classification.

\begin{tabular}{lll}
\hline \hline & \multicolumn{2}{c}{ Predictions $\hat{f}(x)$} \\
\cline { 2 - 3 } Observations & +1 & -1 \\
\hline+1 & TP & FP \\
-1 & FN & TN \\
\hline \hline
\end{tabular}

method is the partial least squares discriminant analysis (PLS-DA) ${ }^{30}$ classification model. One of the key steps in using this method is to extract the set of model components, which describes the maximum correlation between the data of each day. This is achieved by cross-validation, which consists of randomly separating across all daily reflection rate of plants into two parts: learning with $1 / 3$ of the database and testing with $2 / 3$ of the remaining, in order to recalculate the model and identify the smallest set of main components to perform the least squares discriminant analysis on these components, rather than on the initial data.

In order to judge the performance of classification, we calculate a confusion matrix that allows us to collect the results of the classification model. It contains in rows the observations $y$, and in columns the predictions $\hat{f}(x)$ as shown in Table 3. The elements of the matrix represent the number of data corresponding to each case.

The result of a model can be one of four possibilities:

$$
\left\{\begin{array}{ll}
\hat{f}\left(x_{i}\right)=+1 \text { et } y_{i}=+1 & \text { true positive }(\mathrm{TP}) \\
\hat{f}\left(x_{i}\right)=+1 \text { et } y_{i}=-1 & \text { false positive }(\mathrm{FP}) \\
\hat{f}\left(x_{i}\right)=-1 \text { et } y_{i}=-1 & \text { true negative }(\mathrm{TN}) \\
\hat{f}\left(x_{i}\right)=-1 \text { et } y_{i}=+1 & \text { false negative }(\mathrm{FN})
\end{array} .\right.
$$

An error-free model will have results concentrated on the diagonal of its confusion matrix (TP and TN). The accuracy $P$ of the model can be calculated from the confusion matrix as follows:

$$
P=\frac{\mathrm{TP}+\mathrm{TN}}{\mathrm{TP}+\mathrm{FP}+\mathrm{TN}+\mathrm{FN}}
$$

Table 4 groups confusion matrix and accuracy for 11 days of the experiment, obtained during the learning and validation of the PLS-DA model. It can be seen that for the first day of measurement, the data are not linearly separable, e.g., according to confusion matrix of day 1, all leaves of class +1 (control) were properly classified. For class -1 (stressed), four leaves were well classified and six leaves were misclassified. 
Table 4 PLS-DA classification model performance during 11 days of experiment.

\begin{tabular}{|c|c|c|c|c|}
\hline \multicolumn{5}{|c|}{ Confusion matrix } \\
\hline Day 1 & Day 2 & Day 3 & & Day 4 \\
\hline$\left(\begin{array}{cc}10 & 6 \\
0 & 4\end{array}\right)$ & $\left(\begin{array}{ll}8 & 2 \\
2 & 8\end{array}\right)$ & $\left(\begin{array}{cc}10 & 4 \\
0 & 6\end{array}\right)$ & & $\left(\begin{array}{ll}9 & 2 \\
1 & 8\end{array}\right)$ \\
\hline P: $70 \%$ & P: $\mathbf{8 0 \%}$ & P: $\mathbf{8 0 \%}$ & & P: $85 \%$ \\
\hline Day 5 & Day 6 & Day 7 & & Day 8 \\
\hline$\left(\begin{array}{ll}9 & 1 \\
1 & 9\end{array}\right)$ & $\left(\begin{array}{cc}10 & 1 \\
0 & 9\end{array}\right)$ & $\left(\begin{array}{cc}10 & 0 \\
0 & 10\end{array}\right)$ & & $\left(\begin{array}{cc}10 & 0 \\
0 & 10\end{array}\right)$ \\
\hline P: $90 \%$ & P: $95 \%$ & P: $100 \%$ & & P: $100 \%$ \\
\hline Day 9 & Day 10 & & Day 11 & \\
\hline$\left(\begin{array}{cc}10 & 0 \\
0 & 10\end{array}\right)$ & $\left(\begin{array}{cc}10 & 0 \\
0 & 10\end{array}\right)$ & & $\left(\begin{array}{cc}10 & 0 \\
0 & 10\end{array}\right)$ & \\
\hline P: $100 \%$ & P: $100 \%$ & & P: $100 \%$ & \\
\hline
\end{tabular}

Figure 7 presents the accuracy of the model prediction for each day. We note that in the first day of the stress protocol, the model can separate our two classes at $70 \%$, and at $100 \%$ after the 7th day, which suggests that an early detection of water stress would be possible before it can be visible on the plant (day 11).

The analysis tools of the constructed classification model are scores and regression coefficients (loadings). We present these scores in Fig 8(a) plotted from the first two PLS components. The dots in blue represent leaves from controlled plants and in red leaves from the stressed plants. We note that with PLS-DA model constructed, it appears to be possible to separate two classes of plants from 6th day. We also note that until the end of the experiment (11th day), the two classes of plants remain well separated.

Figure 8(b) shows a graphical representation of PLS coefficients that automatically distinguish between control and stressed plants according to the constructed PLS-DA model. The regression coefficients obtained by the PLS-DA model allow us to estimate the linear relationship between PLS components and leaf reflection rate measurements. The coefficients closer to 0 are generally insignificant. The evaluation of significant regression coefficients involves selecting wavelengths sensitive to water stress.

Figure 8 suggests that during all days of measurements, the spectral range between 3.9 and $4.7 \mu \mathrm{m}$ has the strongest influence on the separation of water status because contains high values of PLS coefficients. We also detect some significant wavelengths in spectral range between 7.5

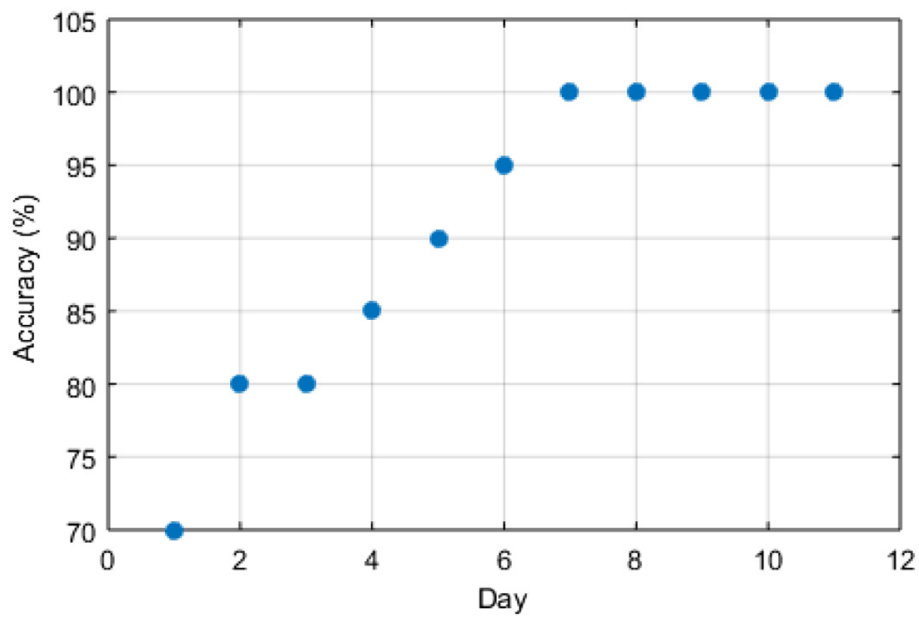

Fig. 7 Accuracy of classification model for each measurement day. 
El Fakir et al.: Active hyperspectral mid-infrared imaging based on a widely tunable quantum...
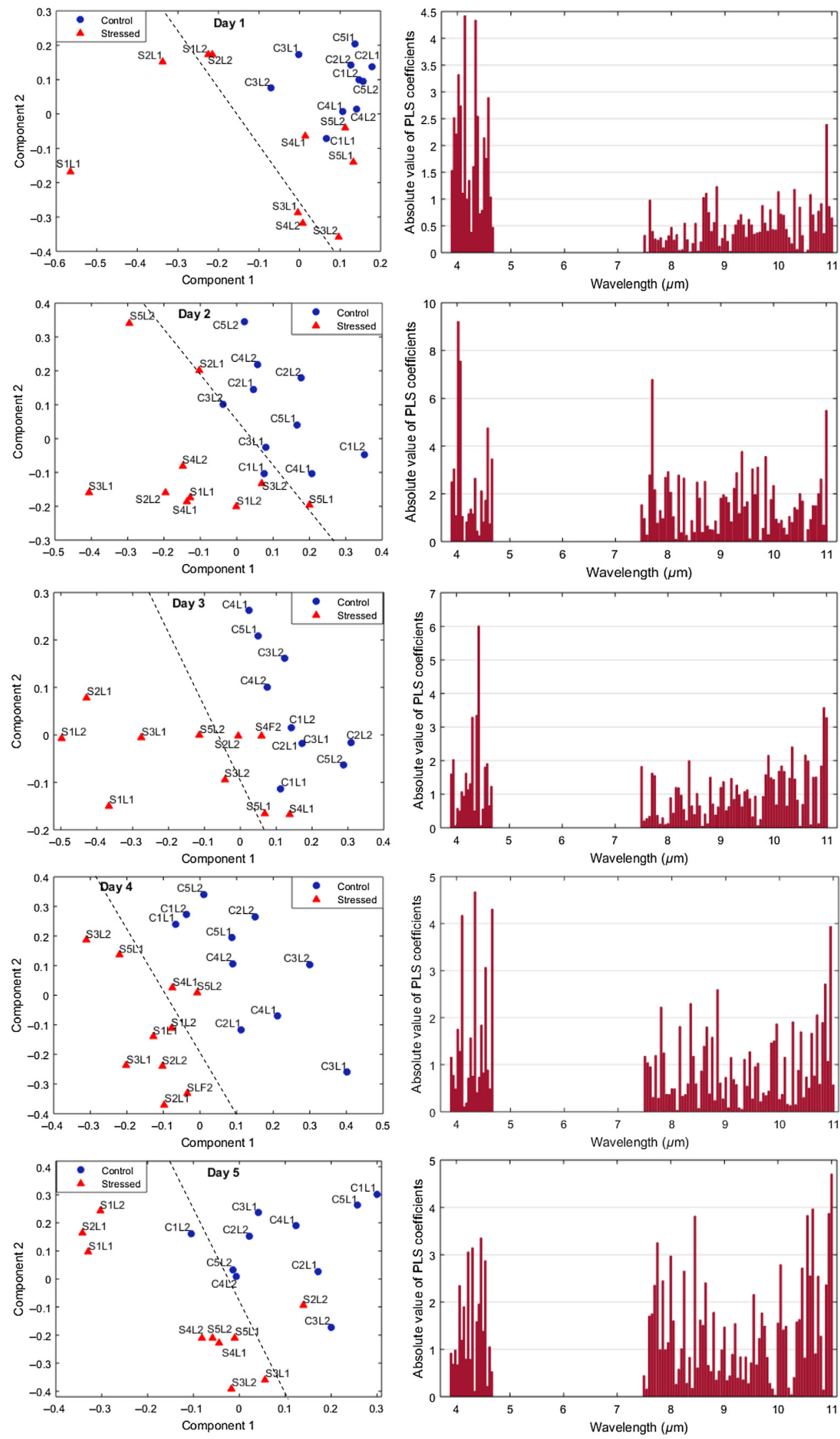

Fig. 8 PLS-DA classification results for 11 days of measurements: (a) scores with labels S: stressed, C: control, and L: leaf and (b) loading associated to wavelength. 
El Fakir et al.: Active hyperspectral mid-infrared imaging based on a widely tunable quantum...
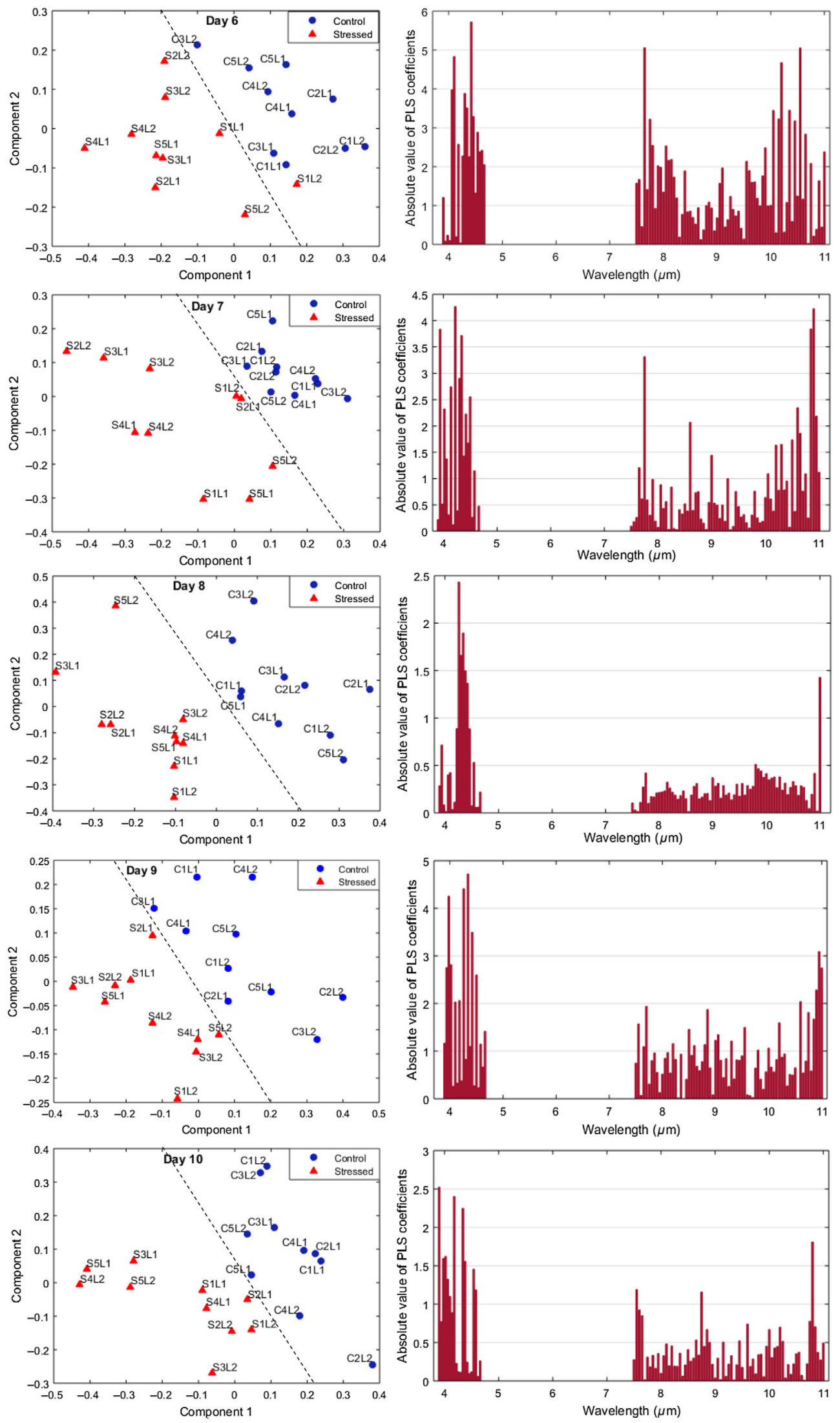

Fig. 8 (Continued.) 


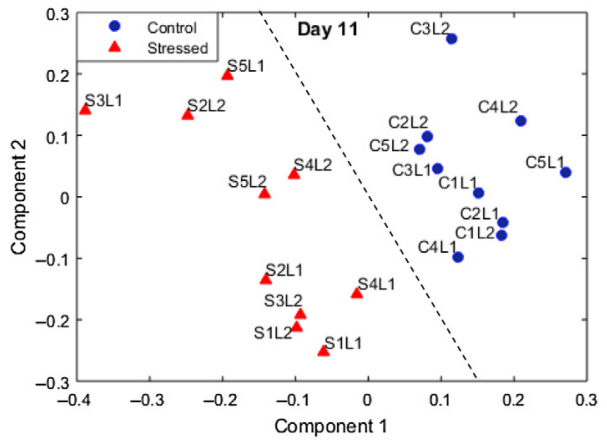

(a)

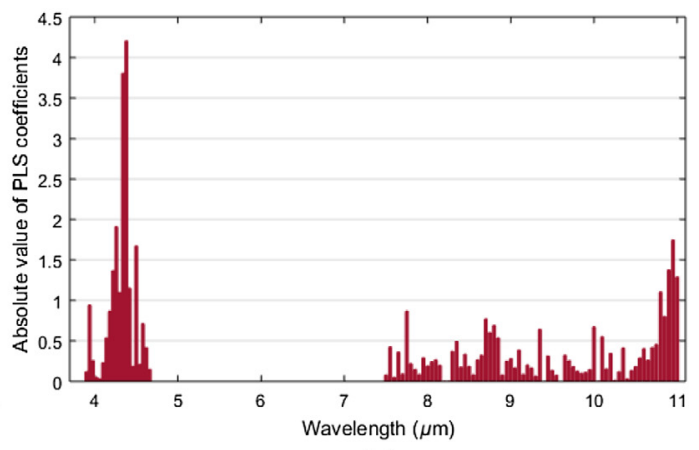

(b)

Fig. 8 (Continued.)

and $11 \mu \mathrm{m}$ such as: $7.75,8,8.45,10.05,10.2,10.55,10.85,10.9 \mu \mathrm{m}$, which means that this spectral region also contributes to characterize water stress of these plants.

\section{Conclusion}

We propose an active hyperspectral imaging system, based on a "staring" architecture, which consists of a widely tunable monochromatic laser that illuminates a scene without any spectral filter and collects the radiation scattered by a broadband camera.

The characteristics of this hyperspectral imaging system are:

- two spectral ranges: from 3 to $5 \mu \mathrm{m}$ and from 7.5 to $11 \mu \mathrm{m}$;

- $20 \mathrm{~cm}^{2}$ image area;

- image capture at a distance of more than $1 \mathrm{~m}$ from the scene.

This system was used to characterize water stress of plants growing in the laboratory. Two water regimes were used: normal water supply corresponding to $80 \%$ SWC and reduced water supply to $20 \%$ SWC.

Since no obvious spectral variation can be observed while applying stress, a machine learning method is proposed to classify the plants according their water stress status, after applying spatial average on images. The database was processed using PLS-DA method for the 11 days of measurements. The graphical representation of the scores showed the ability to separate the two categories of plants from the sixth day of experiment and confirmed the possibility to characterize plants under water stress in these spectral ranges. The regression coefficients allowed the selection of spectral regions that influence this classification. These promising results can be further developed in future studies for other types of stress on plants (biotic or abiotic) or used for investigations of the physical properties of samples in the MIR range.

\section{Acknowledgements}

The authors would like to thank the Roullier Group, especially the Global Innovation Center located in Saint-Malo (France) for funding this research as well as for the plants they provided us with to carry out the measurements.

\section{References}

1. L. Jasper, "How plants sense and respond to stressful environments," Plant Physiol. 182(4), 1624-1635 (2020).

2. T. Gasper, "Concepts in plant stress physiology. Application to plant tissue cultures," Plant Growth Regul. 37(3), 263-285 (2002).

3. L. Chaerle and D. V. Straeten, "Seeing is believing: imaging techniques to monitor plant health," Biochim Biophys Acta 1519(3), 153-166 (2001). 
4. Z. K. Zovko, "Hyperspectral remote sensing of grapevine drought stress," Precis. Agric. 20, 335-347 (2019).

5. R. Shortt et al., "Monitoring soil moisture to improve irrigation decisions," Ministry of Agriculture, Food and Rural Affairs, Ontario, AGDEX 753/560 (2011).

6. S. Fabre, "Influence of water content on spectral reflectance of leaves in the 3-15 $\mu \mathrm{m}$ domain," IEEE Geosci. Remote Sens. Lett. 8, 143-147 (2011).

7. D. C. Brainard et al., "Managing drought risk in a changing climate irrigation and cultivar impacts on Michigan asparagus," Agric. Water Manage. 213, 773-781 (2019).

8. K. Dahal et al., "Improving potato stress tolerance and tuber yield under a climate change scenario. A current overview," Front Plant Sci. 10, 563 (2019).

9. B. Bayat et al., "Remote sensing of grass response to drought stress using spectroscopic techniques and canopy reflectance model inversion," Remote Sens. 8(7), 557 (2016).

10. J. Kunz et al., "Rapid detection of drought stress in plants using femtosecond laser induced breakdown spectroscopy," Opt. Express 25(7), 7251-7262 (2017).

11. J. Behman, J. Steinrücken, and L. Plümer, "Detection of early plant stress responses in hyperspectral images," ISPRS J. Photogramm. Remote Sens. 93, 98-111 (2014).

12. M. Wahabzada et al., "Metro maps of plant disease dynamics automated mining of differences using hyperspectral images," PLoS One 10(1), e0116902 (2015).

13. P. Mishra et al., "Close range hyperspectral imaging of plants: a review," Biosyst. Eng. 164, 49-67 (2017).

14. M. Maimaitiyiming et al., "Early detection of plant physiological responses to different levels of water stress using reflectance spectroscopy," Remote Sens. 9(7), 745 (2017).

15. D. A Sims and J. A. Gamon, "Relationships between leaf pigment content and spectral reflectance across a wide range of species, leaf structures and developmental stages," Remote Sens. Environ. 81(2-3), 337-354 (2002).

16. E. B. Knipling, "Physical and physiological basis for the reflectance of visible and nearinfrared radiation from vegetation," Remote Sens. Environ. 1, 155-159 (1970).

17. S. Stagakis et al., "Monitoring water stress and fruit quality in an orange orchard under regulated deficit irrigation using narrow-band structural and physiological remote sensing indices," ISPRS J. Photogramm. Remote Sens. 71, 47-61 (2012).

18. H. Feng et al., "Modified shortwave infrared perpendicular water stress index: a farmland water stress monitoring method," J. Appl. Meteorol. Climatol. 52, 2024-2032 (2013).

19. M. F. Buitrago et al., "Spectroscopic determination of leaf traits using infrared spectra," Int. J. Appl. Earth Obs. Geoinf. 69, 237-250 (2018).

20. M. F. Buitrago et al., "Changes in thermal infrared spectra of plants caused by temperature and water stress," ISPRS J. Photogramm. Remote Sens. 111, 22-31 (2016).

21. M. P. Diago et al., "Assessment of grapevine water status from hyperspectral imaging of leaves," in Acta Horticult. (ISHS), pp. 89-96 (2014).

22. J. Huang et al., "Hyperspectral detection of rice damaged by rice leaf folder (Cnaphalocrocis medinalis)," Comput. Electron. Agric. 82(March), 100-107 (2012).

23. C. Baccani et al., "Optical design of a hyperspectral drone advanced camera for soil monitoring using an electro-optical liquid crystal technology," Proc. SPIE 10690, 106900P (2018).

24. R. Nasi et al., "Remote sensing of bark beetle damage in urban forests at individual tree level using a novel hyperspectral camera from UAV and aircraft," Urban For. Urban Green. 30, 72-83 (2018).

25. A. K. Goyal et al., "Laser-based long-wave-infrared hyperspectral imaging system for the standoff detection of trace surface chemicals," Opt. Eng. 59(9), 092003 (2020).

26. L. Qingli et al., "Review of spectral imaging technology in biomedical engineering: achievements and challenges," J. Biomed. Opt. 18(10), 100901 (2013).

27. C. El Fakir et al., "Active hyperspectral mid-infrared imaging based on widely tunable QCL laser," in 21st Int. Conf. Transparent Opt. Networks ICTON, Angers, France, IEEE (2019).

28. M. G. Piles et al., "Towards improved spatio-temporel resolution soil moisture retrievals from the synergy of SMOS and MSG SEVIRI spaceborne observations," Remote Sens. Environ. 180, 403-417 (2016). 
29. G. P. Petropoulos et al., "Evaluating the capabilities of optical/TIR imaging sensing systems for quantifying soil water content," Geocarto Int. 35, 494-511 (2018).

30. H. Abdi, "Partial least squares (PLS) regression," in Encyclopedia of Social Sciences Research Methods, M. Lewis-Beck, A. Bryman, and T. Futing, Eds., Vol. 6, No. 4, pp. 792 795, Sage, Thousand Oaks (2003).

Chaimae El Fakir received her PhD in photonics in 2020 from CNRS Foton Institue, Lannion, France. She joined CNRS IMS, Bordeaux, France, in October 2020. Her current research focuses on multispectral imaging in mid-infrared range to localize the signature of specific structural defects in temperature and luminescence of semiconductor components.

Maroun Hjeij graduated with his master's degree in physics and instrumentation in 2019 from Western Brittany University. He is currently working in research at CNRS Foton Institute, Lannion, France, as a $\mathrm{PhD}$ candidate. His current research interests are in the area of hyperspectral imaging systems using quantum cascade lasers for agricultural applications.

Ronan Le Page received his PhD in electronics in 2003. He joined CNRS Foton Institute, Lannion, France, in 2005 as research engineer. Current research mainly deals with signal and image processing, embedded systems, and machine learning in photonics.

Luiz Poffo received his $\mathrm{PhD}$ in photonics in 2007. He has been an associate professor since 2009 at Foton Institut, Lannion, France. His research focuses on integrated optics sensors, photonics sensor for health, food and agriculture applications, and hyper-spectral imaging.

Bastien Billiot received his $\mathrm{PhD}$ in computer science (image analysis) in 2013 and is now incharge of computer vision and statistics research at Agro Innovation International. His research focuses on computer vision, remote sensing, machine learning, and deep learning applied to plant phenotyping (shoot and root part).

Pascal Besnard received his PhD in laser physics from the University of Rennes 1 in $1991 . \mathrm{He}$ spent 1 year as a postdoctoral researcher at the Ontario Lightwave and Laser Research Center, Toronto, Canada. He is a professor of photonics at ENSSAT. He is the head of the Foton Institute and a deputy director of ENSSAT. His principal research interests include laser physics, photonics using semiconductor, and fiber technology for optical communications, imaging and sensors.

Jean-Marc Goujon graduated his PhD in optoelectronics in 1996. Since 1998, he has been an associate professor at CNRS Foton Institute, Lannion, France. His current research interest lie in photonics sensor systems for health, food, and agricultural applications, focusing on infrared and imaging spectral systems. 